\title{
Comunicación
}

\section{Otitis externa canina por Aspergillus niger patogénico: reporte de caso}

\author{
Canine otitis externa by pathogenic Aspergillus niger: case report
}

Gabriela Ygreda ${ }^{1}$, Roy Andrade ${ }^{2}$, Luis M. Jara ${ }^{2,3}$

\section{Resumen}

El presente artículo reporta un caso clínico de otomicosis por Aspergillus en un Cocker Spaniel Inglés macho de 8 años, con diagnóstico previo de anemia hemolítica inmunomediada. El paciente fue atendido por presentar dolor e incomodidad en ambos oídos. Se realizaron análisis sanguíneos, además de citología y cultivo microbiológico de secreción ótica. El Aspergillus niger junto con Malassezia pachydermatis fueron los agentes aislados, para lo cual se instauró una terapia antifúngica oral y tópica con resultados favorables después de 30 días de tratamiento. El moho aislado presentó algunos factores de virulencia importantes como actividad hemolítica, lipasa y amilasa, además de susceptibilidad in vitro a antifúngicos del tipo azoles. En pacientes inmunosuprimidos que presenten otitis externa es necesario realizar cultivos microbiológicos para hongos ambientales, además de evaluar a nivel fenotípico la característica patógena, lo cual ayude a establecer un correcto abordaje terapéutico y monitoreo de enzimas hepáticas por posibles efectos adversos.

Palabras clave: otitis externa canina, Aspergillus niger, inmunosupresión, otomicosis

\footnotetext{
${ }^{1}$ Clínica Veterinaria Docente Cayetano Heredia, Facultad de Medicina Veterinaria y Zootecnia, Universidad Peruana Cayetano Heredia, Lima, Perú

${ }^{2}$ Laboratorio de Patología Clínica, Facultad de Medicina Veterinaria y Zootecnia, Universidad Peruana Cayetano Heredia, Lima, Perú

${ }^{3}$ E-mail: luis.jara.s@upch.pe
}

Recibido: 26 de noviembre de 2020

Aceptado para publicación: 14 de mayo de 2021

Publicado: 24 de agosto de 2021

CLos autores. Este artículo es publicado por la Rev Inv Vet Perú de la Facultad de Medicina Veterinaria, Universidad Nacional Mayor de San Marcos. Este es un artículo de acceso abierto, distribuido bajo los términos de la licencia Creative Commons Atribución 4.0 Internacional (CC BY 4.0) [https:// creativecommons.org/licenses/by/4.0/deed.es] que permite el uso, distribución y reproducción en cualquier medio, siempre que la obra original sea debidamente citada de su fuente original 
This paper reports a clinical case of Aspergillus otomycosis in an 8-year-old male English Cocker Spaniel, with a previous diagnosis of immune-mediated hemolytic anemia. The patient was treated for pain and discomfort in both ears. Routine blood tests were performed, as well as cytology and microbiological culture of ear secretion. Aspergillus niger together with Malassezia pachydermatis were the isolated, the oral and topical antifungal therapy was established with favorable results after 30 days of treatment. The isolated mold exhibited some important virulence factors such as hemolytic, lipase and amylase activity, as well as in vitro susceptibility to azole antifungals. In the immunosuppressed patients with otitis externa, it is necessary to perform microbiological cultures for environmental fungi, in addition to evaluating the pathogenic characteristic at the phenotypic level. This may help to establish a proper therapeutic approach and monitoring of liver enzymes for possible adverse effects.

Key words: canine otitis externa, Aspergillus niger, immunosuppression, otomycosis

\section{INTRODUCCIÓN}

La otitis es una de las afecciones más comunes en perros, presentándose en alrededor del $20 \%$ de las consultas médicas (Angus, 2004). La otitis externa se caracteriza por la inflamación del canal auditivo con diversos grados de eritema en el pabellón, meato y canal auditivo externo. Las bacterias y levaduras patógenas más comunes asociadas suelen ser Staphylococcus sp y Malassezia sp (principalmente $M$. pachydermatis, M. furfur y $M$. obtusa) (Crespo et al., 2000). Otro tipo de bacterias pueden estar presentes de forma normal en el canal auditivo, las cuales pueden volverse patógenas debido a una proliferación excesiva. En comparación a hongos filamentosos, existen pocos reportes de infecciones óticas $\mathrm{u}$ otomicosis, por ejemplo, Aspergillus niger y A. flavus (Goodale et al., 2016).

La aspergilosis es una micosis causada por hongos patógenos del género Aspergillus. Estos corresponden a un grupo de hongos saprofíticos ambientales que ocasionalmente causan infecciones oportunistas en humanos y pequeños animales (Payne et al., 2017). Producen una variedad de formas de enfermedad que pueden ser no invasivas, semi- invasivas o invasivas en perros y gatos (Sykes, 2014). Se incluyen la queratomicosis, otitis externa fúngica, aspergilosis sinonasal, sinoorbital, broncopulmonar y diseminada (Schultz et al., 2008; Coyner, 2010).

En caninos y felinos se presentan principalmente tres formas de aspergilosis: nasal, pulmonar e infecciones diseminadas. $\mathrm{La}$ aspergilosis nasal presenta frecuentemente sinusitis invasiva, ocasionada por las especies $A$. flavus, A. fumigatus y $A$. niger (Ballber et al., 2018; Belda et al., 2018). La de tipo pulmonar es de rara presentación y es causada por similares especies, además de $A$. versicolor (Maniam et al., 2017), mientras que la forma diseminada es una infección infrecuente, pero potencialmente fatal en perros, siendo las especies más aisladas A. terreus, A. deflectus y A. caninus (Kano et al., 2019; Yang et al., 2019), seguido en menor frecuencia por A. fumigatus (Brocal et al., 2019).

El presente reporte describe un caso poco frecuente de aspergilosis ótica en un canino asociado a un proceso inmunosupresor. Además, se presentan las características patogénicas del agente aislado y la evolución del tratamiento instaurado. 

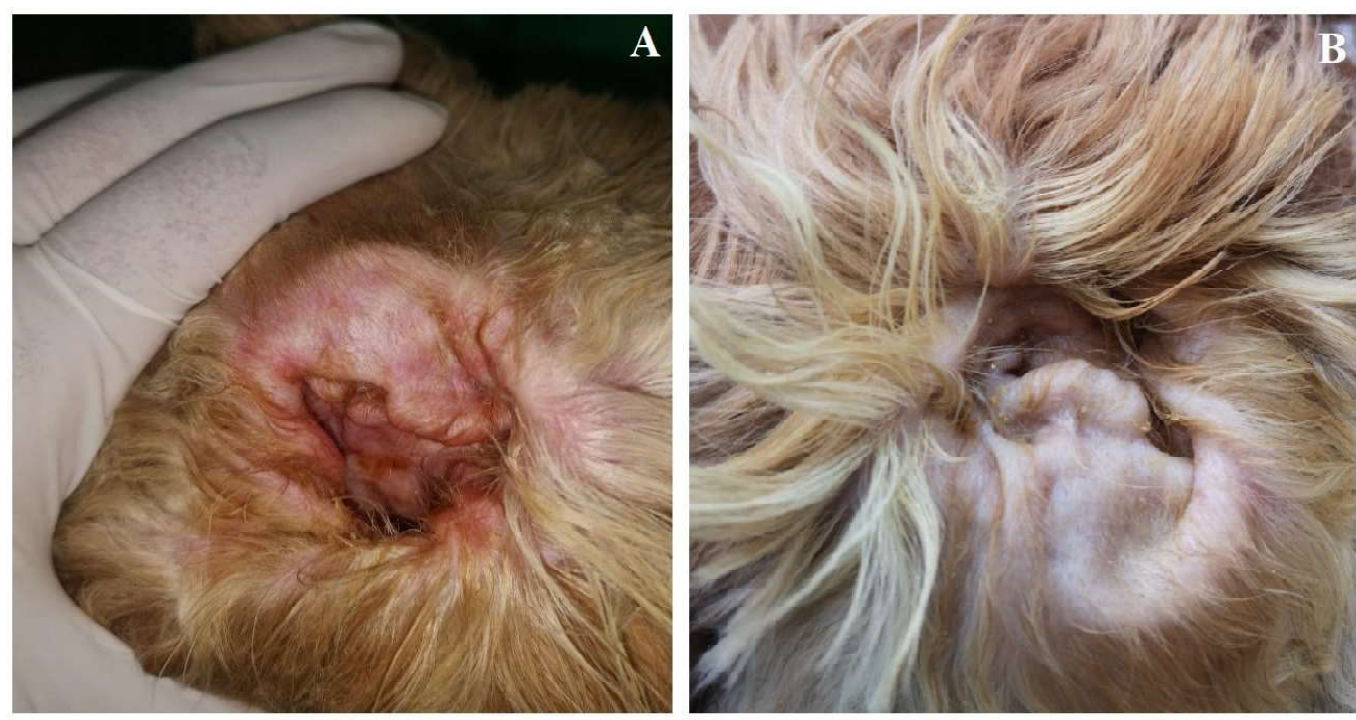

Figura. 1. Oído derecho antes (A) y después (B) del tratamiento con antifúngicos orales y tópicos

\section{Caso Clínico}

Canino macho, Cocker Spaniel Inglés de 8 años de edad, es llevado a consulta de medicina interna a inicios de 2020 en la Clínica Veterinaria Docente Cayetano Heredia, Lima, Perú. Como antecedente se indica que seis meses atrás se le realizó una ecografía donde se encontró hepatomegalia, colecistitis y esplenomegalia con zonas de aparente infarto y necrosis, además de una prueba de Coombs directo con resultado positivo. El diagnóstico fue compatible con anemia hemolítica autoinmune, por lo que se optó por una esplenectomía total. El tratamiento pos-quirúrgico consistió en $0.5 \mathrm{mg} / \mathrm{kg}$ de prednisona, $500 \mathrm{mg}$ de silimarina, $250 \mathrm{mg}$ de ácido ursodeoxicólico y $10 \mathrm{mg} / \mathrm{kg}$ de doxiciclina cada 24 horas por 15 días. Posteriormente se cambió la medicación inmunosupresora por azatioprina a una dosis de $2 \mathrm{mg} / \mathrm{kg}$ una vez al día.

El paciente presentaba un mes antes de la consulta sacudidas frecuentes de cabeza, prurito, dolor e incomodidad en ambos oídos, siendo mayor en el derecho. Al examen físico se registró una condición corporal $4 / 5$, mucosas rosadas y piel de aspecto normal sin presencia de lesiones. Se evidenció eritema e inflamación en ambos pabellones auriculares, además de secreción ótica amarillenta, levemente sanguinolenta. Dichos hallazgos fueron más acentuados en el oído derecho (Figura 1A). No se encontró comprometido el oído medio ni se observó ruptura de tímpano.

Se realizaron análisis sanguíneos y citología, así como cultivo de bacterias y hongos junto con un antibiograma a partir de las secreciones óticas. En el hemograma no se encontraron mayores alteraciones a excepción de policromasia, hematíes nucleados y anisocitosis. De igual forma en el perfil bioquímico sérico solo se halló hiperproteinemia e hiperglobulinemia $(9.0$ y $5.7 \mathrm{~g} / \mathrm{dl}$, respectivamente). En la citología de ambos oídos se observaron escasos leucocitos, abundantes células epiteliales y estructuras ovoides sugerentes de Malassezia sp. 

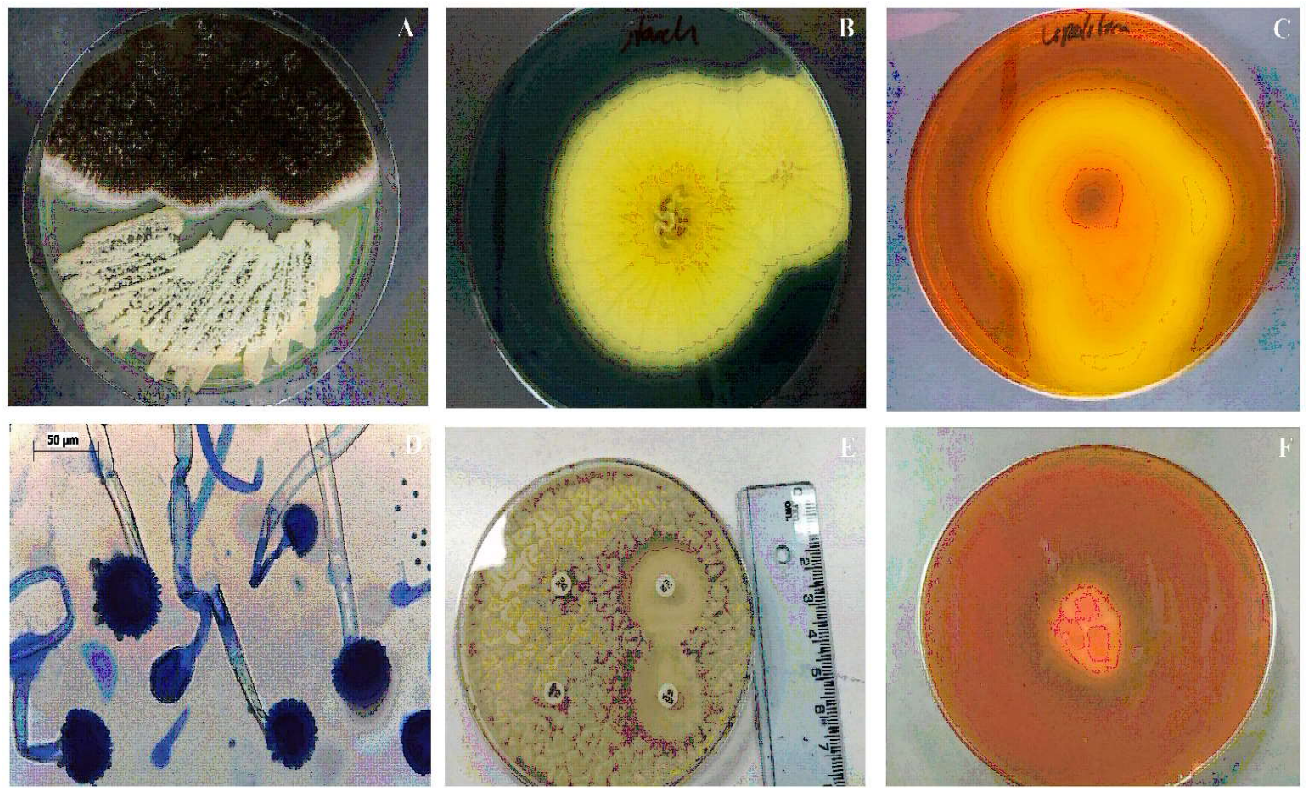

Figura. 2. Características fenotípicas de Aspergillus niger aislado de oído derecho de un perro Cocker Spaniel Inglés de 8 años. A) A. niger en agar Sabourad dextrosa; B) Actividad amilolítica en agar almidón; C) Actividad lipolítica en agar polisobarto 80-rojo fenol; D) Conidias de A. niger, técnica de cinta scotch con tinción azul de lactofenol; E) Antifungigrama mediante difusión en agar; F) Actividad hemolítica completa en agar sangre de carnero al 5\%

El cultivo de bacterias en agar MacConkey y sangre de carnero desfibrinada al $5 \%$ resultó negativo a las 48 horas. Sin embargo, a las 24 horas de cultivo en agar Sabouraud dextrosa se encontró $M$. pachydermatis en la muestra de oído izquierdo y derecho, mientras que se observó crecimiento de Aspergillus sp en el oído derecho. Dicho hongo filamentoso fue identificado como $A$. niger por sus características macroscópicas y microscópicas (Figura 2A-D). La cepa fúngica tuvo actividad hemolítica completa en agar sangre (Figura 2F), lipolítica en agar con polisorbato 80-rojo fenol (Figura 2C) y amilolítica en agar almidón (Figura 2B) (Raksha et al., 2017). Asimismo, resultó resistente frente a discos con antifúngicos como anfotericina B $(100 \mu \mathrm{g})$, fluconazol $(25 \mu \mathrm{g}) \mathrm{y}$ griseofulvina $(10 \mu \mathrm{g})$, en comparación a la susceptibilidad intermedia hallada para miconazol $(10 \mu \mathrm{g})$ y sensibilidad para ketoconazol $(10 \mu \mathrm{g})$, mediante la metodología de difusión en agar (Figura 2E) (Tokarzewski, 2012).

La terapia antifúngica consistió en $5 \mathrm{mg} /$ $\mathrm{kg}$ de itraconazol y $500 \mathrm{mg}$ de silimarina vía oral cada 24 horas por 30 días, además de clorhexidina $(0.5 \mathrm{~g})$, ciprofloxacina $(0.8 \mathrm{~g})$, ketoconazol $(2 \mathrm{~g})$ y prednisolona $(0.25 \mathrm{~g})$ vía topical en los oídos afectados por 45 días. Sesenta días después de la terapia, se realizó un cultivo de hongos del oído afectado y un perfil bioquímico sérico de control, encontrándose negativo para Aspergillus, elevación moderada de alanina aminotransferasa (ALT, $236 \mathrm{U} /$ 1), fosfatasa alcalina (ALP, 165 U/1) y mínima de aspartato aminotransferasa (AST, $44 \mathrm{U} / 1$ ). 
Se observó abundante presencia de cerumen y leve inflamación en ambos oídos, además de engrosamiento del canal auditivo izquierdo. La propietaria mencionó que el tratamiento tópico fue aplicado de manera intermitente. A pesar de que las molestias disminuyeron y no se presentó sintomatología adicional, se realizó una citología de ambos oídos donde se encontraron cocos Gram positivos, células epiteliales y leucocitos. En el cultivo se registró la presencia de $M$. pachydermatis y Staphylococcus sp multirresistente y susceptible a carbapenémicos.

Se modificó el tratamiento con aplicación de limpiador ótico con escualeno al $25 \%$ junto con una receta magistral a base de amikacina $(14 \mathrm{mg} / \mathrm{ml})$, dexametasona $(0.9 \mathrm{mg} / \mathrm{ml})$ y solución salina; que se aplicó una hora después de la limpieza. Un mes después se realizó un nuevo control citológico, encontrándose escasa cantidad de estructuras levaduriformes, sin presencia de bacterias o hifas. Se observó mejoría y disminución de la inflamación en los oídos (Figura 1B). Una semana después el paciente presentó cuadros de hematemesis, hematoquecia, decaimiento, anorexia, trombocitopenia (plaquetas: $<50000$ ) y anemia severa (hematocrito 9.7\%); motivo que provocó el fallecimiento.

\section{Discusión}

La otitis externa es una alteración común en animales de compañía, siendo más frecuente en algunas razas. En un estudio de casos de otitis crónica el $60 \%$ correspondió a los Cocker Spaniel, los que pueden presentar una mayor incidencia de causas primarias que contribuyen a una respuesta severa al estímulo inflamatorio (Angus et al., 2002), mientras que el agente fúngico más frecuentemente reportado es Malassezia sp (Ghibaudo y Peano, 2010), ya que en perros clínicamente sanos puede actuar como un saprofito (Crespo et al., 2002).
Se sabe que la aspergilosis en animales no es una patología frecuente; sin embargo, puede ocurrir en pacientes sanos bajo situaciones de estrés o en condiciones de inmunosupresión iatrogénica o endógena, produciendo desde infecciones localizadas hasta diseminadas, así como reacciones alérgicas (Coyner, 2010; Goodale et al., 2016; Seyedmousavi, 2019). Los pacientes inmunosuprimidos son más susceptibles a una infección diseminada hacia otros órganos como hígado, corazón, páncreas, pulmones (Sykes, 2014), por lo que es recomendable el tratamiento inmediato con medicación antifúngica vía oral y tópica.

En el manejo de la anemia hemolítica autoinmune existe la posibilidad de infecciones oportunistas al administrar medicamentos inmunosupresores en dosis altas o en combinaciones, solo o en combinación con glucocorticoides. Se incluyen infecciones fúngicas o bacterianas del tracto urinario, nocardiosis diseminada y lesiones inducidas por virus del papiloma, aunque no se ha determinado la frecuencia de estas complicaciones (Swann y Skelly, 2016). Sumado a ello, esta enfermedad es considerada de mal pronóstico en canes, con tasas de mortalidad del 30 al 40\% (Goggs et al., 2015). En el presente reporte no se pudo determinar mediante una necropsia las posibles causas del fallecimiento del paciente; sin embargo, en humanos se han reportado fallas renales agudas y multiorgánicas en casos severos (Fattizzo et al., 2020).

La patogenia del Aspergillus depende de varios factores como estar presente en altas concentraciones en el medio ambiente, crecer más rápido que cualquier otro hongo, como lo evidenciado en el presente reporte con el crecimiento luego de $24 \mathrm{~h}$, y superar las defensas del hospedero, sobre todo si existe una inmunidad baja (Tekaia y Latgé, 2005). Se han identificado además algunos factores de virulencia putativos para diferentes especies de Aspergillus. Estos incluyen, por ejemplo, los que generan la degradación de los 
carbohidratos tisulares ( $\alpha$-amilasa), proteínas (proteinasa), fosfolípidos (fosfolipasa), pectinas (pectinasa), lípidos (lipasa) o lisis de glóbulos rojos (hemolisina). Las toxinas además causan inicialmente un trastorno de la función celular, progresando gradualmente a necrosis y lisis celular que finalmente pueden conducir a una falla orgánica (Tomee y Kauffman, 2000). La detección de factores de virulencia puede por tanto ayudar a diferenciar cepas patógenas, además de indicar cuando una terapia con medicamentos antimicóticos es necesaria (Raksha et al., 2017).

Es de importancia destacar la citología ótica como técnica práctica para identificar y caracterizar preliminarmente a microorganismos o alteraciones. En el caso de aspergilosis, es posible observar las hifas características de la especie involucrada. No obstante, para la identificación definitiva de las especies, es necesario aplicar técnicas moleculares, ya que las citologías o cultivos fúngicos pueden ser subjetivas en algunas especies similares, además de presentar baja sensibilidad (Sykes, 2014).

Asimismo, es importante realizar un examen de susceptibilidad in vitro a drogas antifúngicas, aunque aún no se ha determinado los valores referenciales para las concentraciones mínimas inhibitorias en medicina veterinaria. Para el tratamiento frente a Aspergillus sp se recomienda, tanto para humanos como para animales, los fármacos provenientes de las familias de los polienos y azoles (Seyedmousavi, 2019). Sin embargo, tal como lo obtenido en este caso clínico, se sabe que algunas drogas como el fluconazol son resistentes para Aspergillus sp por lo que no está recomendado. Esto se evidenció en el estudio de Goodale et al. (2016) donde del total de pacientes con otitis por Aspergillus sp y tratados con fluconazol, solo el $75 \%$ mostró mejoría, mientras que la infección fue resuelta en el 100\% de aquellos que recibie- ron itraconazol. Además, a diferencia de otros antifúngicos pertenecientes a la familia de los azoles, el itraconazol es uno de los más seguros y con menos efectos adversos (Papich, 2016).

Para casos de otitis externa, el tratamiento de elección se puede administrar vía tópica, por lo que se puede hacer uso de recetas magistrales; mientras que un tratamiento sistémico solo debe ser usado en caso el epitelio del canal auditivo se encuentre erosionado o ulcerado (Pye, 2018). En caso de otitis media, los tratamientos sistémicos son efectivos, debido a la vascularidad de la zona (Morris, 2004). Es importante mencionar que el uso de carbapenémicos en otitis bacterianas, especialmente meropenem, está recomendado en caso de otitis media multirresistente (Morris, 2004).

Es recomendable, además, realizar un monitoreo de enzimas hepáticas antes y después del tratamiento con ketoconazol $\mathrm{u}$ otros azoles, ya que pueden ocurrir reacciones adversas como: hiporexia, pérdida de peso, vómitos e ictericia, así como un incremento de enzimas hepáticas como ALT, ALP e hiperbilirrubinemia (Macho et al., 2020). Elevaciones en ALT y AST reflejarían hepatitis, aunque la elevación en suero de isoenzimas de ALP puede deberse también a la coexistencia de inflamación o infección sistémica, como en el caso de otitis o dermatitis moderadas a severas, además de posible coadministración de glucocorticoides (Center, 2002. Cuando se tienen algunos de estos hallazgos, se debe reducir la dosis o interrumpir el tratamiento hasta una reducción gradual de las enzimas hepáticas. En humanos, la reducción hasta valores normales puede demorar un promedio de 7 semanas luego de la interrupción del tratamiento (Tverdek et al., 2016). Esto se evidenció en el presente caso, donde 30 días después de la interrupción del tratamiento aún se observó la elevación de ALP y ALT. 


\section{ConClusiones}

Condiciones inmunosupresoras pueden estar relacionadas con la presencia de hongos ambientales como Aspergillus patogénico en otitis externa canina. La evaluación fenotípica in vitro de factores de virulencia del hongo es importante para diferenciar la característica potencial patógena asociada a la sintomatología clínica. La terapia antifúngica debe considerar el tratamiento oral y tópico con triazoles, seguido de un monitoreo de las enzimas hepáticas.

\section{Literatura Citada}

1. Angus JC. 2004. Otic cytology in health and disease. Vet Clin North Am Small Anim Pract 34: 411-424. doi: 10.1016/ j.cvsm.2003.10.005

2. Angus JC, Lichtensteiger C, Campbell KL, Schaeffer DJ. 2002. Breed variations in histopathologic features of chronic severe otitis externa in dogs: 80 cases (1995-2001). J Am Vet Med Assoc 221: 1000-1006. doi: 10.2460/ javma.2002.221.1000

3. Ballber C, Hill TL, Bommer NX. 2018. Minimally invasive treatment of sino-nasal aspergillosis in dogs. J Vet Intern Med 32: 2069-2073. doi: 10.1111/ jvim. 15311

4. Belda B, Petrovich N, Mathews KG. 2018. Sinonasal aspergillosis: outcome after topical treatment in dogs with cribriform plate lysis. J Vet Intern Med 32: 1353-1358. doi: 10.1111/jvim.15219.

5. Brocal J, Ribas Del Río F, FeliuPascual AL. 2019. Diagnosis and management of lumbar Aspergillus spp. discospondylitis using intraoperative cytology and external stabilization in a dog with disseminated infection. Open Vet J 9: 185-189. doi: 10.4314/ovj.v9i3.1

6. Center SA. 2007. Interpretation of liver enzymes. Vet Clin North Am Small Anim Prac 37: 297-333. doi: 10.1016/j.cvsm.2006.11.009
7. Coyner K. 2010. Otomycosis due to Aspergillus spp. in a dog: case report and literature review. Vet Dermatol 21: 613-618. doi: 10.1111/j.13653164.2010.00903.x

8. Crespo MJ, Abarca ML, Cabañes FJ. 2002. Occurrence of Malassezia spp. in the external ear canals of dogs and cats with and without otitis externa. Med Mycol 40: 115-121. doi: 10.1080/ mmy.40.2.115.121

9. Fattizzo B, Giannotta JA, Serpenti F, Barcellini $W$. 2020. Difficult cases of autoimmune hemolytic anemia: a challenge for the internal medicine specialist. J Clin Med 9: 3858. doi: 10.3390/jcm9123858

10. Ghibaudo G, Peano A. 2010. Chronic monolateral otomycosis in a dog caused by Aspergillus ochraceus: otomycosis in a dog. Vet Dermatol 21: 522-526. doi: 10.1111/j.1365-3164.2010.00884.x

11. Goggs R, Dennis SG, Di Bella A, Humm KR, McLauchlan G, Mooney $C$, et al. 2015. Predicting outcome in dogs with primary immune-mediated hemolytic anemia: results of a multicenter case registry. J Vet Intern Med 29: 1603 1610. doi: $10.1111 /$ jvim. 13642

12. Goodale E, Outerbridge CA, White $S D .2016$. Aspergillus otitis in small animals - a retrospective study of 17 cases. Vet Dermatol 27: 3-e2. doi: 10.1111/vde. 12283

13. Kano R, Sakai M, Hiyama M, Tani K. 2019. Isolation of Aspergillus caninus (Synonym: Phialosimplex caninus) from a canine iliac lymph node. Mycopathologia 184: 335-339. doi: 10.1007/s11046-018-0312-3

14. Macho LP, Center SA, Randolph JF, Dumars LA, Rush SE, Cameron MK, et al. 2020. Clinical, clinicopathologic, and hepatic histopathologic features associated with probable ketoconazoleinduced liver injury in dogs: 15 cases (2015-2018). J Am Vet Med Assoc 256: 1245-1256. doi: 10.2460/javma.256.11.1245 
15. Maniam R, Selvarajah GT, Mazlan M, Lung Than LT. 2017. Pulmonary papillary adenocarcinoma with Aspergillus versicolor infection in a dog. Med Mycol Case Rep 19: 25-29. doi: 10.1016/ j.mmcr.2017.11.005

16. Morris DO. 2004. Medical therapy of otitis externa and otitis media. Vet Clin North Am Small Anim Pract 34: 541-555. doi: 10.1016/j.cvsm.2003.10.009

17. Papich MG 2016. Saunders handbook of veterinary drugs. $4^{\text {th }}$ ed. St. Louis, MO: WB Saunders. 928 p.

18. Payne CL, Dark MJ, Conway JA, Farina $L L$. 2017. A retrospective study of the prevalence of calcium oxalate crystals in veterinary Aspergillus cases. J Vet Diagn Invest 29: 51-58. doi: 10.1177/1040638716672254

19. Pye C. 2018. Pseudomonas otitis externa in dogs. Can Vet J 59: 1231-1234.

20. Raksha, Singh G, Urhekar AD. 2017. Virulence factors detection in Aspergillus isolates from clinical and environmental samples. J Clin Diagn Res 11: DC13-DC18. doi: 10.7860/JCDR/2017/ 24055.10211

21. Schultz RM, Johnson EG, Wisner ER, Brown NA, Byrne BA, Sykes JE. 2008. Clinicopathologic and diagnostic imaging characteristics of systemic aspergillosis in 30 dogs. J Vet Intern Med 22: 851-859. doi: 10.1111/j.1939-1676.2008.0125.x

22. Seyedmousavi S. 2019. Aspergillosis in humans and animals. In Singh K, Srivastava N. (eds). Recent trends in human and animal mycology. Singapore: Springer. p 81-98.

23. Swann JW, Skelly BJ. 2016. Canine autoimmune hemolytic anemia: management challenges. Vet Med 7: 101112. doi: $10.2147 /$ VMRR.S81869

24. Tekaia F, Latgé JP. 2005. Aspergillus fumigatus: saprophyte or pathogen? Curr Opin Microbiol 8: 385-392. doi: 10.1016/j.mib.2005.06.017

25. Sykes JE. 2014. Aspergillosis. In: Canine and feline infectious diseases. Elsevier. p 633-648.

26. Tokarzewski S, Zión ${ }^{3}$ kowska G, Nowakiewicz A. 2012. Susceptibility testing of Aspergillus niger strains isolated from poultry to antifungal drugs - a comparative study of the disk diffusion, broth microdilution (M38-A) and Etest methods. Pol J Vet Sci 15: 125133. doi: 10.2478/v10181-011-0123-7

27. Tomee, JF, Kauffman HF. 2000. Putative virulence factors of Aspergillus fumigatus. Clin Exp Allergy 30: 476-484. doi: 10.1046/j.1365-2222.2000.00796.x

28. Tverdek FP, Kofteridis D, Kontoyiannis DP. 2016. Antifungal agents and liver toxicity: a complex interaction. Expert Rev Anti Infect Ther 14: 765-776. doi: 10.1080/147872-10.2016.1199272

29. Yang W, Jones BR, Rossi G, Stephens $N$, Arthur I, Merritt A, Reese S, Langner K. 2019. First case of a dog infected with Aspergillus (Phialosimplex) caninus in Australasia. N Z Vet J 68: 231-237. doi: 10.1080/00480169.2019.1706659 